\title{
Elucidating the clinical-pathological factors and prognosis in breast cancer young surviving women who had a subsequent pregnant
}

\author{
Ramírez-Torres Nicolás ${ }^{1}$, Hernández-Valencia Marcelino 2, * and Rivas-Ruíz Rodolfo ${ }^{3}$ \\ ${ }^{1}$ Oncological Gynecology Service, Hospital of Gynecology and Obstetrics No. 3, National Medical Center (NMC) La Raza, \\ Mexican Institute of Social Security (IMSS), Mexico City, Mexico. \\ 2 Medical Research Unit in Endocrine Diseases, Hospital of Specialities, NMC SXXI, IMSS, Mexico City, Mexico. \\ ${ }^{3}$ Clinical Research Training Center, Health Research Coordination, NMC SXXI, IMSS, Mexico City, Mexico.
}

International Journal of Biological and Pharmaceutical Sciences Archive, 2021, 02(02), 013-025

Publication history: Received on 12 September 2021; revised on 29 October 2021; accepted on 31 October 2021

Article DOI: https://doi.org/10.53771/ijbpsa.2021.2.2.0090

\begin{abstract}
Objective. To elucidate the impact of clinical-pathological factors on overall survival (OS) in patients who got pregnant after breast cancer treatment.

Methods. Retrospective cohort of women age younger than 40 years with breast cancer history without active disease at diagnosis of postcancer pregnancy. Clinical-pathological factors were analized by age group and recent birth. Overall survival (OS) was evaluated from Kaplan-Meier method. The association between clinical-pathological factors and OS was examined using Cox proportional hazards method to estimate hazard ratio (HR) with $95 \%$ confidence intervals (CI).
\end{abstract}

Results: A total of 14 patients were selected. Median age was 28.5 years (interquartile range, 26-35). Locally advanced stage (IIB-IIIB) was diagnosed in $64.3 \%$. Patients lower than 35 years experienced more positive clinical lymph nodes (72.7\%), grade $2(63.6 \%)$ and ER/PgR-negative tumors (54.5\% and $72.7 \%$, respectively). The patients with ER-positive tumors showed an improvement non-significant at 5-year OS $(87 \% ; p=0.097)$.

In the bivariate analysis, patients with a higher number of pathological lymph nodes (pNs) had a $12 \%$ increase in the risk of death than those with lower number ( $\mathrm{HR}=1.12 ; 95 \% \mathrm{CI}: 1.02$ to 1.2). The multivariate model (after adjustment for number of pNs, age and tumor size) ascertained that the nodal status was the only independent predictor associated to a worse OS (HR $=1.15$; $95 \% \mathrm{CI}: 1.01$ to 1.3 ).

Conclusion. Pregnancy after cancer did not have a detrimental effect on survival. The patients $<35$ years old group showed more unfavorable tumor features at diagnosis, which can largely explain a poorer prognosis. Nodal status was the most important prognostic factor that predicted the poor prognosis.

Keywords: Breast cancer; Young age; Subsequent pregnancy' Postpartum period; Surviving.

\section{Background}

Breast cancer (BC) usually affects women at perimenopause and postmenopausal age; approximately $7 \%$ to $16.2 \%$ of all $\mathrm{BC}$ cases are diagnosed in women younger than 40 years [1-5]. The number of cases tends to increase as our

\footnotetext{
* Corresponding author: Hernández-Valencia Marcelino

Medical Research Unit in Endocrine Diseases, Hospital of Specialities, NMC SXXI, IMSS, Mexico City, Mexico. 
population grows $[1,2]$. Women age younger than 45 years who have survived following $\mathrm{BC}$ treatment have been able to conceive 6-22 with low pregnancy rates $(3 \%$ to $8 \%)[8-13,15,20]$.

Extensive research has been addressed at determining the prognostic impact of women who became pregnant after BC diagnosis and treatment, evaluating whether mortality varies in relation to time of diagnosis during pregnancy and in different postpartum periods and also whether mortality varies over time from diagnosis $[6,7,12,17,18]$.

Many studies have pointed that women with a post-cancer pregnancy appear to have a better prognosis compared with those did not [6-17]. However, many BC surviving women were older than 30 years and had not initiated childbearing. Several studies have revealed that young women are more likely to develop unfavorable tumor histopathological features and more aggressive subtypes [1-5].

Clinical and pathological factors in premenopausal patients $<40$ years old with primary BC have been evaluated [1-5, 22]. It is not clear whether the dismal results seen in young women $<40$ years old are a reflection of advanced stage at diagnosis or due to differences in the underlying tumor biology [1-5].

This study determines prognostic value of clinical and histopathological factors, outcomes of overall survival (OS) of women who got pregnant with BC history and the impact of subsequent pregnancy on breast cancer.

\section{Patients and methods}

\subsection{Study population}

This study examines an observational retrospective cohort of patients who achieved to become pregnant after BC treatment, the study started in March 1994 and the follow-up was complemented in June 2010 at Hospital of Gynecology and Obstetrics No. 3, NMC "La Raza" of the Mexican Institute of Social Security.

We included premenopausal women age 40 years or younger with history of treated BC followed by a subsequent pregnancy, with diagnosis of unilateral BC, clinical stage I, II and III, completed oncological treatment before pregnancy, and absence of local and distant disease at pregnancy diagnosis with at least one year elapsed after the conclusion of chemotherapy. Histological diagnosis of invasive BC and molecular diagnosis were obtained of tissue biopsies of the primary tumor.

Complete BC staging was performed in all patients. The following imaging studies according to the clinical stage were carried out: mammography, liver ultrasound, and radiographic skeletal survey, which included chest X-ray. BC was classified according to tumor-node-metastasis (TNM) clinical staging from the American Joint Committee on Cancer/ The International Union Against Cancer (AJCC/ UICC), 6th edition [23]. TNM stage established prior to the year 2002 was reclassified.

Subsequent pregnancy after breast cancer (SPBC) is defined as the pregnancy occurring at least one year after BC diagnosis and treatment [7] without local or distant disease evidence at pregnancy diagnosis. Some authors have defined the postpartum period as the time interval ranging from 6 months to 2 years after delivery $[6,18]$.

\subsection{Clinical and pathological factors}

Data were obtained from medical records. The following tumor features were included: primary tumor size, axillary lymph node (N), histology, histological grade (HG), lymphovascular invasion (LVI) and pathological lymph nodes (pNs).

Biological markers by immunohistochemistry (IHC) were determined for estrogen receptor (ER), progesterone receptor (PgR) and human epidermal growth factor receptor 2 (HER2). It was also taken into account the Nottingham prognostic index (NPI).

Since no IHC material was available for Ki67, BC molecular subtype was approximated according to grade and IHC surrogates (ER, PgR and HER2) to categorize five clinical subtypes: luminal A (LA), luminal B/HER2-negative (LB/HER), luminal B/HER2-positive (LB/HER+), HER2-positive (HER2+) and triple negative (TN) [ 4, 24].

\subsection{Treatment}


A multimodal oncological treatment was the standard of care according to local protocols in those days at our institution. Detailed oncological treatment information (surgery, radiotherapy, neoadjuvant chemotherapy [NC] and adjuvant chemotherapy $[\mathrm{AC}]$ ), as well as obstetrical and neonatal data were collected previously in a retrospective study [22].

We mainly used a 5-fluorouracil, epirubicin and cyclophosphamide (FEC)-based chemotherapy both for NC and AC. All procedures were conducted with informed consent from patients in accordance with the ethical standards of institutional research committee and national laws, their names were omitted from the database.

\subsection{Statistical analysis}

The primary objective was to evaluate the impact of clinical-pathological factors on OS. The secondary objective was to calculate survival curves in accordance to clinical-pathological factors. The frequencies and percentages were summarized in a contingence table. The prognostic factors were evaluated by age group and by time relapsed between $\mathrm{BC}$ diagnosis and recent birth, the groups were compared using the Fisher's exact test. The main outcomes examined were mortality rate and overall survival (OS).

Asymmetric quantitative data (age, tumor size and NPI) were reported as medians with interquartile ranges (IQR) and were compared using the non-parametric tests. The patient's age was assessed both as categorical variable and quantitative variable.

Kaplan-Meier product limit method was used to assess the survival curves of clinical-pathological factors and log-rank statistic was used to test the differences between groups [25]. The disease free survival (DFS) follow-up began from the date of surgery (breast-conserving therapy or mastectomy) to the date of first recurrence (local or distant) or death, whichever comes first. Local recurrence was defined as any tumor activity that returns in the treated ipsilateral breast or in the chest wall anterior region.

OS followp-up began from the date of histological diagnosis to the date of the last control, loss of follow-up or death, whichever occurred first. Only deaths related to BC were taken into account for OS. Survival in the absence of events was censored until the last control. Follow-up was carried out for the first 60 months.

The association of clinical and pathological factors with the OS of the patients with SPBC was assessed using Cox proportional hazards method ${ }^{26}$. The multivariate models were conducted by stepwise adjustment including potentially confounding covariables: age (quantitative), tumor size (quantitative), number of axillary pNs (quantitative), clinical stage (II/ III), HG (1-2/ 3), histological type (non-ductal carcinoma/ ductal carcinoma), ER (positive/ negative), HER2 (negative/ positive) and time elapsed between BC diagnosis and recent birth ( $<24$ months/ $>24$ months).

The hazard ratio (HR), 95\% confidence intervals (CI) and regression coefficient ( $\beta$ ) were reported. Finally, the proportionality assumption for each variable was checked through time dependent variable. The null hypothesis indicates that the clinical-pathological factors have no a prognostic impact.

All the probability values were two sided with a significance level of $5 \%$. HR was considered statistically significant if the 95\% CI thresholds did not include the null value. The database and all statistical analyses were calculated using SPSS 18 (SPSS Inc, Chicago, IL). Graphs were plotted using STATA 14 (College Station, TX).

\section{Results}

\subsection{Characteristics of patients}

A total of 14 consecutive cases with SPBC were selected. We summarized clinical-pathological characteristics at diagnosis in table 1. Median age of the women was 28.5 years (IQR: 26-35). The youngest age at BC diagnosis $(<30$ years) prevailed in $57.2 \%$ of cases, three $(21.4 \%)$ patients had family history of BC. Median of primary tumor size prior to oncological treatment was $4 \mathrm{~cm}$ (IQR: 3-5).

In our cohort the clinical data most frequent at diagnosis were tumors T2 in $71.4 \%$, axillary clinical Ns in $64.3 \%$, infiltrating ductal carcinoma in $71.4 \%$ and grade 2 in $64.3 \%$. Although tumors smaller than $5 \mathrm{~cm}$ (T2) predominated, when $\mathrm{T}$ and $\mathrm{N}$ were associated for BC TNM staging, two thirds of cases were diagnosed in locally advanced stage (IIBIIIB). Table 1. There was not record of cases with inflammatory BC nor pathological complete response after NC. The 
women age younger than 35 years tend to develop tumors of poor prognosis ( $>5.4)$, the median value of NPI was 5.8 (IQR: 5.6-6.8). Figure 1. TN and LA tumors were the most common subtypes (each one in 28.6\%). Figure 2.

\subsection{Tumor characteristics by age group}

The distribution of clinical-pathological factors by age group did not differ significantly. We identified some differences in patients age younger than 35 years group, they experienced more nodes-positive (72.7\%), grade 1-2 (63.6\%), ER/PgR-negative tumors (54.5\% and 72.7\%, respectively) and one third of women showed HER2 overexpression. In contrast, for women age older than 35 years, all tumors were ER/PgR-positive. Table 2.

\subsection{Tumor characteristics by recent birth}

The distribution of clinical-pathological factors in accordance to time elapsed between BC diagnosis and recent birth did not differ significantly, the young women who gave birth within the first 2 years after BC diagnosis showed a higher proportion of younger age (<35 years: 66.6\%), axillary clinical N1-2 (66.6\%), ER-positive (66.6\%) and PgR-negative tumors (55.5\%). One third of cases had HER2 overexpression. Instead, stage IIA-IIB and G1-G2 tumors were the factors more prevalent in both groups without importing for group the date of birth after BC treatment.

\subsection{Survival analysis}

Survival was calculated for each prognostic factor. The patients who had ER-positive tumors than those with ERnegative tumors showed a significant improvement at 5-year DFS (88\% vs.17\%; $p=0.009$ ) but non-significant at 5-year OS (87\% vs. 50\%; $p=0.097)$, respectively. Figures $2 \mathrm{~A}$ and $2 \mathrm{~B}$.

Another important factor is lymph nodal status, patients with pNs $>4$, pNs of 1-3 and pNs-negative showed a 5-year DFS rate of $25 \%, 50 \%$ and $100 \%(p=0.132)$ and a 5 -year OS rate of $50 \%, 75 \%$ and $100 \%(p=0.273)$, respectively. However, the differences were not statistically significant. Figures $2 \mathrm{C}$ and $2 \mathrm{D}$.

The time elapsed between BC diagnosis and recent birth was assessed, the survival at 5 years was different. For DFS was $56 \%$ vs. $60 \%(p=0.777)$ and for OS was $65 \%$ vs. $80 \%(p=0.244)$ in women who gave birth within the first 24 months or beyond 24 months after ending the BC treatment, respectively. But the differences between groups were not statistically significant. Figures $2 \mathrm{E}$ and $2 \mathrm{~F}$. We also assessed other tumor-related factors on DFS and OS, where the differences did not reach statistical significance.

\subsection{Prognosis according to clinical-pathological factors}

The bivariate analysis showed that lymph nodal status was the only independent factor that influenced negatively on OS. The patients who showed a higher number of pNs had a 12\% increase in the risk of death in comparison with those with lower number (crude HR $=1.12 ; 95 \% \mathrm{CI}, 1.02$ to 1.2). In regards to the multivariate model adjusted by number of pNs, age and tumor size, the patients who showed a higher number of pNs had a $15 \%$ increase in the risk of death than those with lower number ( $\mathrm{HR}=1.15 ; 95 \% \mathrm{CI}, 1.01$ to 1.3$)$. Table 4 . The differences for other factors were not statistically significant.

\subsection{Follow-up, recurrences and mortality}

For all cohort, a total analysis at risk of 873 months-person, a DFS mean follow-up of $53 \pm 8$ months (95\% CI: 37.3 to 68.7 ) and OS mean follow-up of $76.5 \pm 8.3$ months (95\% CI: 60 to 92.9) were seen. Six of the 14 patients developed metastatic disease, of which five out of six (83.3\%) were at advanced stage and two out of six (33.3\%) were undifferentiated grade. In regards to subtype, three out of four patients who were HER2-positive tumors (all cases) and three out of four TN tumors developed metastasis. The main metastasis sites in bone and lung (multiple sites: three cases), lung (two cases) and brain (one case) were seen. Locoregional recurrence was associated with systemic disease in three cases (21.4\%) and only two cases (14.2\%) developed local recurrence. All patients with metastatic disease have died. 


\section{Discussion}

Combined oncological treatments have showed an increase in survival rates and women in reproductive age with $\mathrm{BC}$ history also have increased their survival. After BC management, most women probably do not want to initiate childbearing immediately, they face treatment-derived problems [12]. Many young women with BC history without evidence of active disease or recurrence pursue childbearing in the following years, particularly those who do not have children or have not completed their family as planned $[12,20,21,27,28]$.

The gonadotoxic effects of chemotherapy and prolonged endocrine therapy can lead to premature ovarian failure which is a problem for the future fertility [27-29]. The implications of infertility and temporary or permanent amenorrhea after BC treatment are of particular concern in young surviving women [15, 20,29].

In our cohort, it is worth noting that two thirds of women delayed conception. Nine out of 14 patients (64.2\%) were nulliparous at $\mathrm{BC}$ diagnosis, from this group 55.5\% (five out of nine) of women were 30 years of age or older. The mean age of the women who conceived after the BC diagnosis was $31.5 \pm 5$ years old (limits 27-42) and five patients had history of one birth before $\mathrm{BC}$ diagnosis [22].

Clinical and pathological factors help to determine the $\mathrm{BC}$ prognosis. In regards to age, $\mathrm{BC}$ in women age younger than 35 years is characterized by developing unfavorable histopathological parameters, more aggressive phenotypes [1-5, $24]$ and tumors with a poor-prognosis NPI. Several studies have proven that the youngest age is a strong independent predictor associated to a poorer prognosis compared to those older age $[1-5,12,24,28]$. The women with SPBC tend to be younger.

In the regards to molecular subtype, the distribution of $\mathrm{BC}$ subtype differs by race and $\mathrm{TN}$ tumor has higher prevalence among African Americans young patients [2]; younger women tend to develop luminal (A and B) tumors [3-5, 19, 24]. Instead, the study by Azim 30 reported higher incidence of ER-/HER2- tumors among young women.

It is surprising the mortality increase among young women with ER/PgR-positive and LA tumors [3-5, 19, 24], the study by Johansson [24] also assessed the effect of subtype within age group, he found in young women $<39$ years old that the TN and HER2+ subtypes exhibited higher risk of death. Thus, the age and subtype are prognostic factors that have an independent effect on OS [2, 4, 5, 24]. Other studies [31,32] have mentioned certain adverse factors such as resistance to chemotherapy, apoptosis alteration or increased angiogenesis are closely linked with younger age.

In our study, patients at younger age $(<35$ years) showed a poor 5 -year OS of $64 \%(p=0.265)$. When analysing the multivariate model, the young age did not have an independent effect on OS which might be due to lack of statistical power.

The prognosis is poor when the tumor is diagnosed in advanced stages (IIB-IIIB) [1-5, 12, 22, 24], particularly in women age younger than 30 years due to the high expression of unfavorable histological features, which is reflected in significantly lower survival rates and higher local and distant recurrence rates in comparison with older women [1-5].

In the present study, two thirds of young women ( $<40$ years) were diagnosed with locally advanced stage (IIB-IIIB). So that, the women with tumors in stage IIIA-B had a poor 5 -year OS of $60 \%(p=0.823)$, but the difference between groups did not reach statistical significance. In a multivariate model, the stage (IIA-B vs. IIIA-B) contrary to the expected data, the stage did not have an independent effect on DFS or OS.

The $\mathrm{N}$ is most important factor on prognosis. Several studies have evaluated nodal status for patients who became pregnant after BC treatment finding differences in survival. Patients with node-negative disease had better survival rates at 5 years of $76 \%$ to $100 \%[7-9,11,12,15,17$ and 10$]$ years of $86 \%$ to $96 \%[8,11]$ compared to those who had positive nodes, whose survival rates at 5 years were $50 \%$ to $79 \% 8,9$ and 10 years was $79 \%[8,9,12,15]$.

Azim conducted a meta-analysis of 14 studies (1,244 cases and 18,145 controls) to understand the effect of pregnancy on OS of women with history of $\mathrm{BC}$, and he also evaluated the lymph nodal status. The patients with positive nodal disease were more likely to die (pooled relative risk $[\mathrm{PRR}]=0.96(95 \% \mathrm{CI}$ : 0.67 to $1.37 ; \mathrm{I} 2=0)$ than those with negative nodal disease (PRR $=0.63 ; 95 \% \mathrm{CI}: 0.41$ to $0.96 ; \mathrm{I} 2=20.8$ ) [17].

In our study, when we evaluated all pNs subgroups, at least $42.8 \%$ of patients had more than $4 \mathrm{pNs}$ which indicated a poorer prognosis. We were able to confirm in both bivariate and multivariate models that number of pNs was the only independent predictor associated to a worse OS. 
The poor prognosis has been reported for young women with ER-negative tumors $[2,3,5,18]$. Instead, the study by Nagatsuma 19 reported a mortality increase for women with ER-positive tumors in the postpartum period.

In our study, when ER status was stratified by age group, women age younger than 35 years showed a slight predominance of ER-negative disease (54.5\%), which had shorter survival times and an elevated mortality (83.3\%). In the multivariate analysis, ER-negative tumor was not a predictive factor of poor OS among young women.

Young women exhibit more adverse histopathological features; the invasive ductal carcinoma (71.4\% to 88.2\%) [1-5, $19,22]$ and the high-grade tumors are common (24\% to $63.2 \%)[2,3,5,19,24]$, even the grade is part of molecular subtype 24 . LVI accounts for $7 \%$ to $28.6 \%$ of cases 3,22 . The HER2 overexpression confers a more aggressive phenotype at younger age $(24 \%$ to $44.6 \%)[1-5,19,21,22]$, high Ki67 levels are reported $(62.1 \%$ to $71.4 \%) 1,2$ and P53 abnormal expression is less reported (29\%) 3. Thus, the tumor-associated factors among young women, such as age, grade, stage and subtype explain a large part the poor survival compared to screening-aged women [2-5, 24].

Comparative studies such as matched case-control [8, 10-12, 21], population-based [9, 14, 15] and hospital-based studies $[13,16]$ often indicate a good prognosis in most cases and sometimes even better than in women who did not become pregnant. For example, international study by Gelber [11] indicated longer survival times for women who became pregnant in comparison with matched controls: 5-year survival was $92 \%$ vs. $85 \%$ and 10 -year survival was $86 \%$ vs. $74 \%$, respectively. The meta-analysis by Azim [17] reported a $41 \%$ significant reduction in the risk of death for women who got pregnant after BC treatment compared to those who did not (PRR $=0.59 ; 95 \%$ CI: 0.50 to $0.70 ; I^{2}=$ $43.1 \%)$.

In most studies aftermentioned, there was no increased risk of mortality nor high rates of recurrent disease for most patients who became pregnant after BC diagnosis and treatment $[6-17,19,21]$. This relative survival advantage for women with subsequent pregnancies after BC treatment could be reflecting a healthy patient selection bias rather than a true protective effect. These patients often have had long enough survival times, and this way they were able to be included in follow-up studies.

Healthy mothers usually want to conceive after BC diagnosis and treatment. Indeed, most women age younger than 35 years with early disease (smaller tumors and few Ns involved) who are free of active disease or recurrence at the time of conception are more likely to get pregnant after treatment than older women or with disease sequelae [6-22].

Subsequent pregnancy does not seem to affect the survival, but during development of pregnancy there are adverse obstetrical events such as miscarriages, preterm birth (PTB), low birth weight (LBW), fetal anomalies (AF) among other obstetrical complications $[20,29]$. There is a high percentage of induced abortions $(20-69 \%)[11,13,15,17]$.

Gerstl ${ }^{20}$ conducted a meta-analysis of 16 matched cohort and case-control studies (1,287 cases and 22,964 controls), the study is focused on pregnancy outcomes after BC treatment. The surviving women (cases) who had received systemic therapy after surgery showed an estimated pooled pregnancy rate (PPR) of $14 \%$ (95\% CI, 0.12 to $0.16 ; I^{2}=$ $95.4 \%$ ). Of those who became pregnant, $12 \%$ (95\% CI, 0.08 to $0.16 ; I^{2}=65.9 \%$ ) experienced a miscarriage and $21 \%$ (95\% CI, 0.16 to $\left.0.26 ; I^{2}=45.9 \%\right)$ terminated their pregnancy $[10,11,13,20]$. Three population-based studies $(\mathrm{n}=711)$ reported a low PPR of $3 \%(95 \%$ CI, 0.02 to 0.03 ; I2 = 85.1\%) in young women (median age of $31-33)[15,17,20]$.

The meta-analysis by D'Ambrosio ${ }^{28}$ of four studies (1,466 cases and 6,912,485 controls) evaluated the potential risk of obstetrical complications among BC survivors, compared with controls, cases were significantly associated with a higher risk of PTB in 11.05\% (162/1,466), LBW in 9.26\% (119/1,285) and AF in 5.8\% (31/534). The ORs found for PTB, LWB and AF were 1.68 (95\% CI, 1.43 to 1.99; $\left.I^{2}=12 \%\right)$; of 1.88 (95\% CI, 1.55 to 2.27; $I^{2}=0 \%$ ) and 1.45 (95\% CI, 1.01 to $\left.2.09 ; I^{2}=56 \%\right)$, respectively.

Thus, the women with BC history are on average 40\% less likely to get pregnant than the general population [15, 17 , $20]$ and miscarriages are often occurred (10\% to 29\%) [10, 13, 15, 20-22] Women with ER-positive tumors were less likely to get pregnant than women with ER-negative tumors $[11,13,17]$.

In our cohort, binomial control was successfully conducted from the date of pregnancy diagnosis. Seven out of eight term pregnancies $(87.5 \%)$ were ended by caesarean section. The interruption of the preterm pregnancy by caesarean section was due to severe preeclampsia (one case) and two preterm pregnancies had an eutocic delivery; three miscarriages were observed. Apparent phenotypic malformations were not seen and neuropsychological development has been normal in the newborns ${ }^{19}$. 
The subsequent pregnant in women with $\mathrm{BC}$ history does not seem to modify tumor histopathological features nor survival $[6-17,19,20]$. These features in young women with a SPBC did not significantly differ to that of matched controls (non-pregnancy-related breast cancer) by age, stage [6-17,19-21] and race 12 .

A postulate indicates that pregnancy seems to exert a time-dependent dual effect on the risk of breast cancer. It is protective in the long-term, but in the short-term, tumor incidence and aggressiveness are increased [33,34]. There are differences in specific genomic signatures of mammary epithelium between parous and nulliparous women. These genomic signatures identify the degree of mammary gland differentiation induced by pregnancy which is associated with a reduction in the risk of breast cancer [35].

Table 1 Characteristics of patients

\begin{tabular}{|l|l|}
\hline Characteristic & $\mathbf{n}=\mathbf{1 4}(\mathbf{\% )})$ \\
\hline Age, years & \\
Median & 28.5 \\
Interquartile range & $(26-35)$ \\
\hline Age by group & \\
<30 years & $8(57.2)$ \\
31-35 years & $3(21.4)$ \\
$>$ 36 years & $3(21.4)$ \\
\hline Tumor size, cm & \\
Median & 4 \\
IQR & $(3-5)$ \\
\hline Tumor size (T) & \\
T2 & $10(71.4)$ \\
T3 & $2(14.3)$ \\
T4 & $2(14.3)$ \\
\hline Axillary lymph nodes (N) & \\
N0 & $5(35.7)$ \\
N1 & $6(42.9)$ \\
N2 & $3(21.4)$ \\
\hline Clinical stage (TNM) & \\
IIA & $5(35.7)$ \\
IIB-IIIA & $7(50.0)$ \\
IIIB & $2(14.3)$ \\
\hline Histology & \\
Ductal carcinoma & $10(71.4)$ \\
Lobular carcinoma & $2(14.3)$ \\
Medullary carcinoma & $1(7.1)$ \\
Other carcinoma & $1(7.1)$ \\
\hline Histological grade & \\
Grade 1 & $1(7.1)$ \\
Grade 2 & $9(64.3)$ \\
Grade 3 & $4(28.6)$ \\
\hline Lymphovascular invasion & $10(61.4)$ \\
Absent & $4(28.6)$ \\
Present & \\
\hline History of breast cancer & \\
No & \\
\hline
\end{tabular}




\begin{tabular}{|l|l|}
\hline Yes & $3(21.4)$ \\
\hline
\end{tabular}

The hormonal changes and pregnancy-associated immune alterations produce an adverse effect in women who had a birth in a short period (within the first two years) after BC diagnosis [6,11, 15, 18, 33, 34]. Another postulates have indicated that during the process of breast remodeling (involution) after pregnancy is often associated with an increase in the angiogenesis, immune cell influx, inflammation and extracellular matrix alterations [33, 34], producing structural changes in the mammary gland microenvironment, besides may enhance existing malignant cells grow and metastasis $[6,18,33,34]$, especially in patients who have shown HRe-positive disease $[12,15]$.

Some studies have reported an improved survival for patients who delayed conception 24 months or more after BC treatment: increased OS at five years (88.2\% to 100\%). Instead, when women gave birth within the first 24 months after treatment showed a decreased OS at five years (54\% to 85\%) [7, 11, 12, 18, 19]. Thus, the risk of death was more apparent in the patients with a birth in a short period after BC diagnosis, since this women group showed more adverse tumor features $[6,7,12,15,18,19,21]$.

Our data concordant with the aforementioned. In the bivariate analysis, when women delayed conception beyond 24 months had a $72 \%$ reduction in risk of death (crude HR $=0.28 ; 95 \% \mathrm{CI}, 0.03$ to 2.6), but delay of pregnancy after BC diagnosis was not associated to OS. No patient experienced metastasis during development of pregnancy. In regards to subsequent pregnancy after BC diagnosis, this did not adversely affect prognosis as is reported in other studies [6, 8$19,21]$.

This study has some limitations such as retrospective design, the data collection was over a long period and a statistically underpowered sample. Therefore, we had inconsistent results to draw solid inferences on the prognostic value of the different prognostic factors.

Instead, the strength of the study lies in the consistency of standardized oncological therapies for a women group who got pregnant with history of $\mathrm{BC}$ free of disease ${ }^{22}$. Our data are consistent with other studies that have reported tumor features and ultimate prognosis in BC surviving women who had a subsequent pregnancy [6-21].

Although, number of cases is limited, our investigation documents that tumors diagnosed in women age younger than 35 years showed unfavorable tumor features such as invasive ductal carcinoma with spreading toward regional Ns, locally advanced stage (IIB-IIIB), ER/PgR-negative tumors, and a poor-prognosis NPI group, all these findings largely explain why there is a worse survival in this population. These same unfavorable tumor features were found among young women who gave birth within the first 2 years after BC diagnosis, which agrees with other reports $[6,12,18,19$, 21].

Table 2 Clinical and pathological factors by age group

\begin{tabular}{|c|c|c|c|c|}
\hline & & $\begin{array}{l}\text { Age, } \\
<35 \text { years }\end{array}$ & $\begin{array}{l}\text { Age, } \\
>35 \text { years }\end{array}$ & $p^{*}$ \\
\hline Variable & Status & $\mathrm{n}=11(\%)$ & $\mathrm{n}=3(\%)$ & \\
\hline Clinical stage & $\begin{array}{l}\text { IIA-B } \\
\text { IIIA-B }\end{array}$ & $\begin{array}{l}6(54.5) \\
5(45.5)\end{array}$ & $\begin{array}{l}3(100 .) \\
0(0.0)\end{array}$ & 0.258 \\
\hline Lymph nodes & $\begin{array}{l}\text { N0 } \\
\text { N1-N2 }\end{array}$ & $\begin{array}{l}3(27.3) \\
8(72.7)\end{array}$ & $\begin{array}{l}2(66.6) \\
1(33.3) \\
\end{array}$ & 0.207 \\
\hline Tumor grade & $\begin{array}{l}\text { G1-G2 } \\
\text { G3 }\end{array}$ & $\begin{array}{l}7(63.6) \\
4(36.4) \\
\end{array}$ & $\begin{array}{l}3(100 .) \\
0(0.0)\end{array}$ & 0.505 \\
\hline ILV & $\begin{array}{l}\text { Ausent } \\
\text { Present }\end{array}$ & $\begin{array}{l}8(72.7) \\
3(27.3) \\
\end{array}$ & $\begin{array}{l}2(66.6) \\
1(33.4) \\
\end{array}$ & 0.837 \\
\hline ER & $\begin{array}{l}\text { Positive } \\
\text { Negative }\end{array}$ & $\begin{array}{l}5(45.5) \\
6(54.5) \\
\end{array}$ & $\begin{array}{l}3(100 .) \\
0(0.0)\end{array}$ & 0.209 \\
\hline PgR & $\begin{array}{l}\text { Positive } \\
\text { Negative }\end{array}$ & $\begin{array}{l}3(27.3) \\
8(72.7)\end{array}$ & $\begin{array}{l}3(100 .) \\
0(0.0)\end{array}$ & 0.055 \\
\hline
\end{tabular}




\begin{tabular}{|l|l|l|l|l|}
\hline HER2 & Positive & $4(36.4)$ & $0(0.0)$ & 0.505 \\
& Negative & $7(63.6)$ & $3(100)$. & \\
\hline NPI & $<5.4$ & $2(18.1)$ & $3(100)$. & 0.027 \\
& $>5.4$ & $9(81.9)$ & $0(0.0)$ & \\
\hline
\end{tabular}

ER: estrogen receptor; PgR: progesterone receptor; HER2: human epidermal growth factor receptor 2; NPI: Nottingham prognostic index. * Fisher's exact test.

Moreover, adjuvant chemotherapy and adjuvant tamoxifen have achieved to reduce the risk of relapse and have improved both DFS and OS in young BC patients $[2-4,28,36]$ and young age alone should not be the reason to prescribe more aggressive therapies $[28,36]$. Thus, the biological age of the patient should be taken into consideration to establish further researches in this specific field in order to understand better biological differences of the breast cancer in young women and improve medical and psychosocial outcomes, other aspects also should be considered in the young population such as the body image, sexual dysfunction, fertility and the quality of life [17-21, 28, 29, 36].

The women with history of adequately treated BC may have one or more pregnancies. However, there are several reasons to avoid any attempt of conception, such as patient fear of possible relapses during pregnancy, since these are more likely to occur within the first 2 years after BC diagnosis, avoidance both unnecessary miscarriages after treatment and difficult decisions in the management of recurrent disease.

Thus, the best is to individualize, reassure and guide the patients who want to conceive in the future $[11,16,20,21,27$ 29]. When the patient got pregnant, we should conduct a close longitudinal assessment from the first trimester in a maternal-fetal center in order to detect any adverse obstetrical condition [22, 29]. Thus, the subsequent pregnancy is safe in women with history of adequately treated BC.

What is best interval between breast cancer diagnosis and subsequent pregnancy for women who want to get pregnant? Recommendations are to delay pregnancy at least 2 years following BC diagnosis and treatment [12, 20], from 2 to 5 years when the risk of relapse is high after BC therapy and pregnancy is not recommend in patients with recurrent disease in order to avoid motherless children [11,12]. Any alteration should be discussed between a multidisciplinary medical group and the parents.

Table 3 Clinical and pathological factors according to time elapsed between breast cancer diagnosis and recent birth

\begin{tabular}{|c|c|c|c|c|}
\hline & & Birth, < 2 years & Birth, > 2 years & \\
\hline Variable & Status & $\mathrm{n}=9(\%)$ & $\mathrm{n}=5(\%)$ & $\boldsymbol{p}^{*}$ \\
\hline Age, years & $\begin{array}{l}<35 \\
>35\end{array}$ & $\begin{array}{l}6(66.6) \\
3(33.3)\end{array}$ & $\begin{array}{l}5(100 .) \\
0(0.0)\end{array}$ & 0.258 \\
\hline Clinical stage & $\begin{array}{l}\text { IIA-B } \\
\text { IIIA-B }\end{array}$ & $\begin{array}{l}6(66.6) \\
3(33.3)\end{array}$ & $\begin{array}{l}3(60.0) \\
2(30.0)\end{array}$ & 0.803 \\
\hline Lymph nodes & $\begin{array}{l}\text { N0 } \\
\text { N1-N2 }\end{array}$ & $\begin{array}{l}3(33.3) \\
6(66.6)\end{array}$ & $\begin{array}{l}2(40.0) \\
3(60.0)\end{array}$ & 0.803 \\
\hline Tumor grade & $\begin{array}{l}\text { G1-G2 } \\
\text { G3 }\end{array}$ & $\begin{array}{l}7(77.7) \\
2(22.2)\end{array}$ & $\begin{array}{l}3(60.0) \\
2(40.0)\end{array}$ & 0.580 \\
\hline ILV & $\begin{array}{l}\text { Ausent } \\
\text { Present }\end{array}$ & $\begin{array}{l}7(77.7) \\
2(22.2)\end{array}$ & $\begin{array}{l}3(60.0) \\
2(40.0)\end{array}$ & 0.580 \\
\hline ER & $\begin{array}{l}\text { Positive } \\
\text { Negative }\end{array}$ & $\begin{array}{l}6(66.6) \\
3(33.3)\end{array}$ & $\begin{array}{l}2(40.0) \\
3(60.0)\end{array}$ & 0.582 \\
\hline
\end{tabular}


International Journal of Biological and Pharmaceutical Sciences Archive, 2021, 02(02), 013-025

\begin{tabular}{|l|l|l|l|l|}
\hline PgR & $\begin{array}{l}\text { Positive } \\
\text { Negative }\end{array}$ & $\begin{array}{l}4(45.5) \\
5(55.5)\end{array}$ & $\begin{array}{l}2(40.0) \\
3(60.0)\end{array}$ & 0.872 \\
\hline HER2 & Positive & $3(33.3)$ & $1(20.0)$ & 0.597 \\
& Negative & $6(66.6)$ & $4(80.0)$ & 0.581 \\
\hline NPI & $<5.4$ & $4(44.4)$ & $1(20.0)$ & \\
& $>5.4$ & $6(55.6)$ & $4(80.0)$ & \\
\hline
\end{tabular}

ER: estrogen receptor; PgR: progesterone receptor; HER2: human epidermal growth factor receptor $2 .{ }^{*}$ Fisher's exact test.

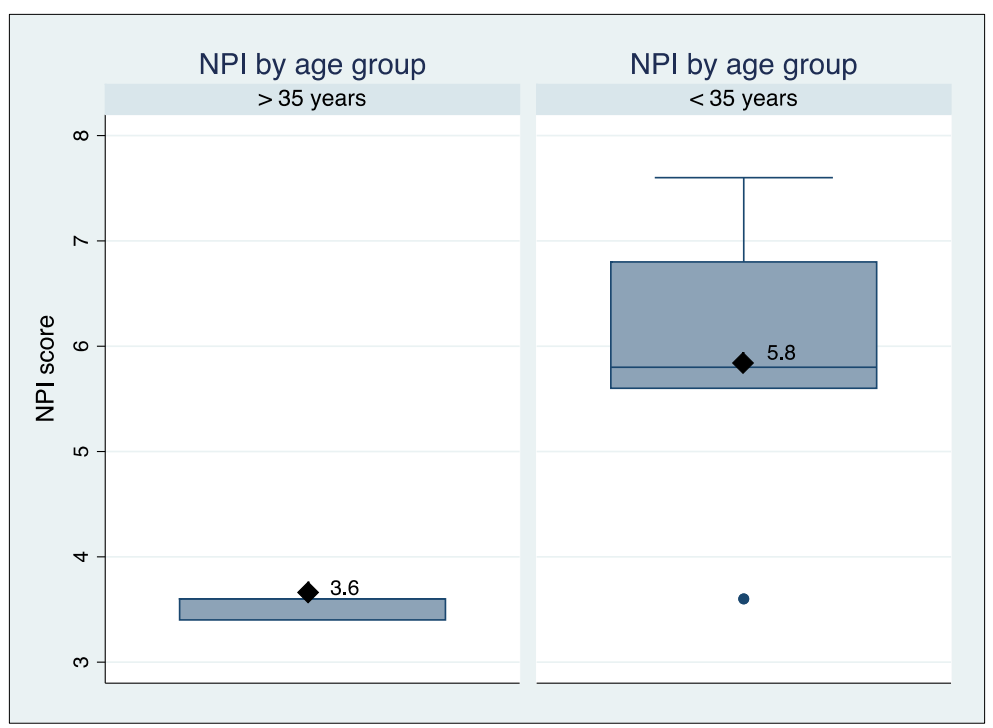

Figure 1 The young women $<35$ years old tend to exhibit poor- prognosis groups.

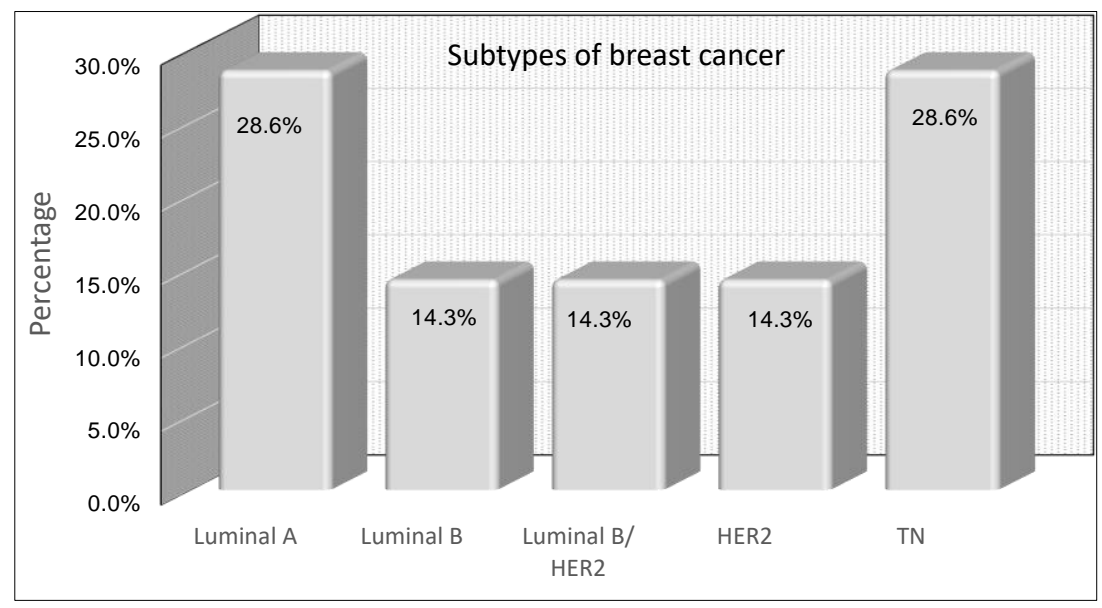

Figure 2 Subtypes of breast cancer in young women who had a subsequent pregnancy after breast cancer treatment.

Table 4 Cox regression analysis of clinical and pathological factors affecting OS in patients with SPBC

\begin{tabular}{|l|l|l|l|l|l|l|l|l|}
\hline & \multicolumn{4}{|l|}{ Bivariate model } & \multicolumn{3}{l|}{ Multivariate model } \\
\hline Variable & $\boldsymbol{\beta}$ & $\mathbf{S E}$ & HR (95\% CI) & $\mathbf{p}$ & $\boldsymbol{\beta}$ & $\mathbf{S E}$ & HR$\left.^{*} \mathbf{( 9 5 \%} \mathbf{C I}\right)$ & $\mathbf{p}$ \\
\hline $\mathrm{pN}$ (number) $\S$ & 0.122 & 0.052 & $1.12(1.02-1.2)$ & 0.019 & 0.140 & 0.066 & $1.15(1.01-1.3)$ & 0.033 \\
\hline
\end{tabular}




\begin{tabular}{|l|l|l|l|l|l|l|l|l|}
\hline Age (years) § & -0.063 & 0.098 & $0.93(0.77-1.1)$ & 0.523 & -0.228 & 0.207 & $0.79(0.53-1.1)$ & 0.271 \\
\hline Tumor $(\mathrm{cm}) \S$ & -0.102 & 0.202 & $0.90(0.60-1.3)$ & 0.616 & -0.352 & 0.352 & $0.70(0.35-1.4)$ & 0.317 \\
\hline
\end{tabular}

SPBC: subsequent pregnancy after breast cancer; OS: overall survival; 目: regression coefficient; SE: standard error; HR: Hazard ratio; CI: confidence interval; §: quantitative variable; pN: pathological lymph node; HR*: adjusted by number of pN, age, and tumor size
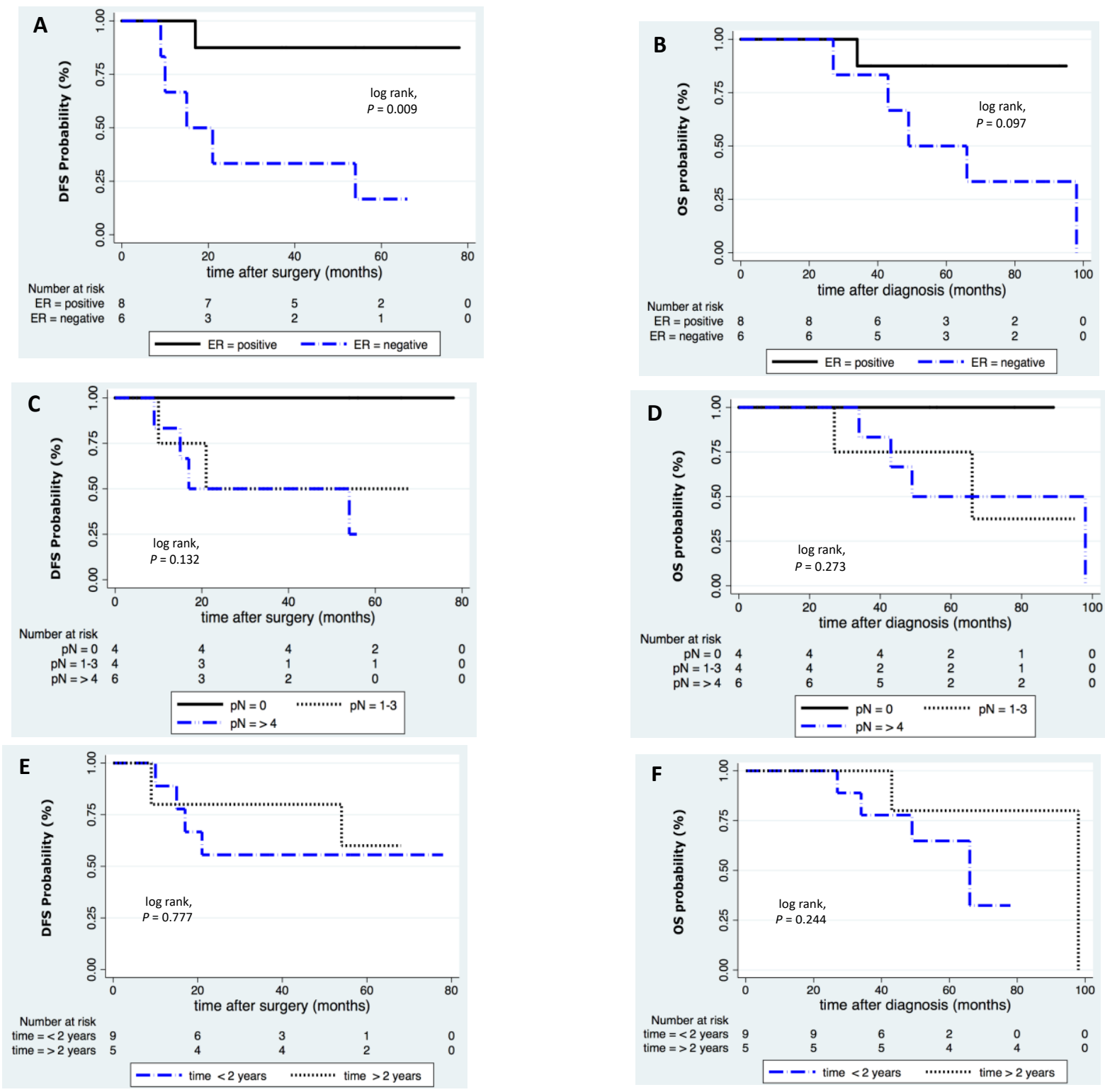

Figure 3 Differences in Kaplan-Meier survival curves among patients with estrogen receptor (ER), pathological lymph nodes (pNs) and time elapsed between breast cancer diagnosis and subsequent pregnancy. Disease free survival (DFS): A, C and E. Overall survival (OS): B, D and F.

\section{Conclusion}

Our study indicates that subsequent pregnancy in the patients who received adequate treatment for BC did not have a detrimental effect on survival nor modify tumor histopathological features. However, younger women $(<35$ years) and those with a recent birth after BC diagnosis were characterized by having more aggressive tumor features. The nodal status seems to indicate that was main prognostic factor associated to poor survival. 
Further efforts should be made to preserve fertility in young women who are willing to consider pregnancy after the completion of chemotherapy. Further researches are needed in order to elucidate the BC biology associated to agespecific tumor features and their relation with multigene expression profiles for this group of patients.

\section{Compliance with ethical standards}

\section{Acknowledgments}

All authors express our gratitude to the patients for sharing their medical history, to social workers Miss Elvira and Miss Sara for administrative support, and librarian Jorge Perez for technical support with the study material. We also express our gratitude to Clinical Research Training Center for statistical support in the development of this study.

\section{Disclosure of conflict of interest}

The authors declare not having any competing interests.

\section{Statement of informed consent}

Informed consent was obtained from all individual participants included in the study.

\section{References}

[1] Hartley M, McKinley B, Rogers EA, et al. Differential expression of prognostic factors and effect on survival in young $(<$ or $=40)$ breast cancer patients. A case control study. Am Surg 2006; 72:1189-94.

[2] Anders CK, Hsu DS, Broadwater G, et al. Young age at diagnosis correlates with worse prognosis and defines a subset of breast cancers with shared patterns of gene expression. J Clin Oncol 2008; 26:3324-30.

[3] van der Hage JA, Mieog JS, van de Velde CJ, et al. Impact of established prognostic factors and molecular subtype in very young breast cancer patients: pooled analysis of four EORTC randomized controlled is i peptrials. Breast Cancer Res 2011; 13:R68.

[4] Loibl S, Jackisch Ch, Lederer B, et al. Outcome after neoadjuvant chemotherapy in young breast cancer patients: a pooled analysis of individual patient data from eight prospectively randomized controlled trials. Breast Cancer Res Treat 2015; 152:377-87.

[5] Partridge AH, Hughes ME, Warner ET, et al. Subtype-dependent relationship between young age at diagnosis and breast cancer survival. J Clin Oncol 2016; 34:3308-14.

[6] Stenheim H, Møller B, van Dijk T, et al. Cause-Specific Survival for women diagnosed with cancer during pregnancy or lactation: A registry-based cohort study. J Clin Oncol 2009; 27:45-51.

[7] Clark RM, Chua T. Breast cancer in pregnancy: the ultimate challenge. Clin Oncol Radiol 1989; 1:11-8.

[8] Sankila R, Heinavaara S, Hakulinen T. Survival of breast cancer patients after subsequent term pregnancy: "Healthy mother effect". Am J Obstet Gynecol 1994; 170:818-23

[9] Lethaby AE, O’Neill MA, Mason BH, et al. Overall survival from breast cancer in women pregnant or lactating at or after diagnosis. Int J Cancer 1996; 67:751-5.

[10] Velentgas P, Daling JR, Malone KE, et al. Pregnancy after breast carcinoma: outcomes and influence on mortality. Cancer 1999; 85:2424-32.

[11] Gelber S, Coates AS, Goldhirsch A, et al. Effect of pregnancy on overall survival after the diagnosis of early-stage breast cancer. J Clin Oncol 2001; 19:1671-5.

[12] Mueller BA, Simen MS, Deapen D, et al. Childbearing and survival after breast carcinoma in young women. Cancer 2003; 98: 1131-40.

[13] Blakely LJ, Buzdar AU, Lozada JA, et al. Effects of pregnancy after treatment for breast carcinoma on survival and risk of recurrence. Cancer 2004; 100:465-9. 
[14] Ives A, Saunders C, Bulsara M, et al. Pregnancy after breast cancer: population based study. BMJ 2007; 334:1948.

[15] Kroman N, Jensen BM, Wohlfahrt J, et al. Pregnancy after treatment of breast cancer - a population-based study on behalf of Danish Breast Cancer Cooperative. Acta Oncol 2008; 47:545-9.

[16] Largillier R, Savignoni A, Glirov J, et al. Prognostic role of pregnancy occurring before or after treatment of early breast cancer. Cancer 2009; 115:5155-65.

[17] Azim HA Jr, Santoro L, Pavlidis N, et al. Safety of pregnancy following breast cancer diagnosis: a meta-analysis of 14 studies. Eur J Cancer 2011; 47:74-83.

[18] Johansson ALV, Andersson TML Hsieh CC, et al. Increased mortality in women with breast cancer detected during pregnancy and different periods postpartum. Cancer Epidemiol Biomarkers Prev 2011; 20:1865-72.

[19] Nagatsuma AK, Shimizu Ch, Takahashi F, et al. Impact of recent parity on histopathological tumor features and breast cancer outcome in premenopausal Japanese women. Breast Cancer Res Treat 2013; 38:941-50.

[20] Gerstl B, Sullivan E, Ives A, et al. Pregnancy outcomes after a breast cancer diagnosis: a systematic review and meta-analysis. Clin Breast Cancer 2018; 18:e79-e88.

[21] Kranick JA, Schaefer C, Rowell S, et al. Is pregnancy after breast cancer safe? Breast J 2010; 16:404-11.

[22] Ramírez-Torres N, Robles-Robles AG, Villafaña-Vázquez VH, et al. Breast cancer and subsequent pregnancy. Infertility, risk of death and survival. Ginecol Obstet Mex 2010; 78:85-93.

[23] Singletary SE, Connolly JL. Breast Cancer Staging: Working with the sixth edition of the AJCC Cancer Staging Manual. CA Cancer J Clin 2006; 56:37-47.

[24] Johansson ALV, Trewin CB, Hjerkind KV, et al. Breast cancer-specific survival by clinical subtype after 7 years follow-up of young and elderly women in a nationwide cohort. Int J Cancer 2019; 144:1251-61.

[25] Clark TG, Bradburn MJ, Love SB, et al. Survival analysis part I: Basic concepts and first analyses. Br J Cancer 2003; 89:232-8.

[26] Pérez-Rodríguez M, Rivas-Ruiz R, Palacios-Cruz L, Talavera JO. Clinical research XXII. From clinical judgment to Cox proportional hazards model. Rev Med Inst Mex Seguro Soc 2014; 52:430-5.

[27] Kopeika J, Bhaduri M, Kugadas A, et al. Planned and unplanned pregnancies in breast cancer survivors. The Breast 2019; 46:75-80.

[28] Paluch-Shimon S, Pagani O, Partridge AH, et al. ESO-ESMO 3rd international consensus guidelines for breast cancer in young women (BCY3). The Breast 2017; 35:203-17.

[29] D’Ambrosio V, Vena F, DiMascio D, et al. Obstetrical outcomes in women with history of breast cáncer: a systematic review and meta-analysis. Breast Cancer Res Treat 2019; 178:485-92.

[30] Azim Jr HA, Michiels S, Bedard P, et al. Elucidating prognosis and biology of breast cancer arising in young women using gene expression profiling. Clin Cancer Res 2012; 18:1241-51.

[31] Holmqvist P, Lundstrÿom M, Stÿal O. Apoptosis and Bcl-2 expression in relation to age, tumor characteristics and

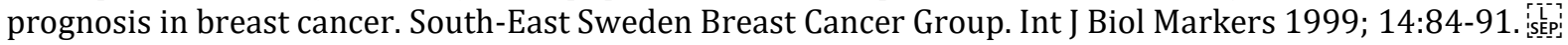

[32] Marinho A, Soares R, Ferro J, et al. Angiogenesis in breast cancer is related to age but not to other prognostic parameters. Pathol Res Pract 1997; 193:267-73.

[33] Lambe M, Hsieh C, Trichopoulos D, et al. Transient increase in the risk of breast cancer after giving birth. $N$ Engl J Med 1994; 331:5-9.

[34] Schedin P, O’Brien J, Rudolph M, et al. Microenvironment of the involuting mammary gland mediates mammary cancer progression. J Mammary Gland Biol Neoplasia 2007; 12:71-82.

[35] Russo J, Balogh GA, Russo IH, et al. Full-term pregnancy induces a specific genomic signature in the human breast. Cancer Epidemiol Biomarkers Prev 2008; 17:51-66.

[36] Early Breast Cancer Trialists' Collaborative G. Effects of chemotherapy and hormonal therapy for early breast cancer on recurrence and 15-year survival: an overview of the randomised trials. Lancet 2005; 365:1687-1717. 